\title{
PAPER
}

\section{Differentiating multiple system atrophy from Parkinson's disease: contribution of striatal and midbrain MRI volumetry and multi-tracer PET imaging}

\author{
M Ghaemi, R Hilker, J Rudolf, J Sobesky, W-D Heiss
}

J Neurol Neurosurg Psychiatry 2002;73:517-523

See end of article for authors' affiliations .....................

Correspondence to: Dr W-D Heiss, Klinik für Neurologie der Universität zu Köln, Joseph-Stelzmann Str 9, 50924 Cologne, Germany;

wdh@pet.mpin-koeln.mpg.de

Received 30 April 2002 In revised form 15 July 2002

Accepted 16 July 2002

\begin{abstract}
Objectives: The differential diagnosis between typical idiopathic Parkinson's disease (PD) and the striatonigral variant of multiple system atrophy (MSA-P) is often difficult because of the presence of signs and symptoms common to both forms of parkinsonism, particularly at symptom onset. This study investigated striatal and midbrain findings in MSA-P and PD patients in comparison with normal controls with the use of positron emission tomography (PET) and three dimensional magnetic resonance imaging (3D MRI) based volumetry to increase the differential diagnostic accuracy between both disease entities.

Methods: Nine patients with MSA-P, 24 patients with PD, and seven healthy controls were studied by MRI and PET with 6-[ $\left[{ }^{18} \mathrm{~F}\right]$-fluoro-L-dopa (FDOPA), [ $\left.{ }^{18} \mathrm{~F}\right]$ fluoro-deoxyglucose (FDG), and 11-C-Raclopride (RACLO). Striatal and extrastriatal volumes of interest (VOI) were calculated on the basis of the individual MRI data. The PET data were transferred to the VOI datasets and subsequently analysed.

Results: MSA-P differed significantly from PD patients in terms of decreased putaminal volume, glucose metabolism, and postsynaptic D2 receptor density. The striatal FDOPA uptake was equally impaired in both conditions. Neither MRI volumetry nor PET imaging of the midbrain region further contributed to the differential diagnosis between PD and MSA-P.

Conclusions: The extent and spatial distribution of functional and morphological changes in the striatum permit the differentiation of MSA-P from PD. Both, multi-tracer PET and 3D MRI based volumetry, may be considered equivalent in the assessment of different striatal abnormality in both disease entities. In contrast, MRI and PET imaging of the midbrain does not provide a further gain in diagnostic accuracy.
\end{abstract}

T he differential diagnosis between idiopathic Parkinson's disease (PD) and parkinsonism predominant multiple system atrophy (MSA-P) is often difficult, particularly at symptom onset. ${ }^{12}$ To find the correct diagnosis early in the course of the disorders is, however, of major importance with respect to prognostic aspects and therapeutic strategies. In particular, MSA-P may present with signs and symptoms in early stages of the disease that are also common to PD. MSA-P was first identified by Adams and colleagues in four patients with severe, progressive parkinsonism in whom postmortem examination disclosed extensive loss of striatal neurons, particularly in the putamen, as well as degeneration of the substantia nigra, ${ }^{3}$ while Lewy bodies, the histopathological hallmark of idiopathic PD, were absent. ${ }^{4}$ Clinically, MSA-P is an akinetic-rigid syndrome characterised by gait disturbance, rigidity, progressive bradykinaesia, and a poor response to dopaminergic therapy.

The identification of patients with clinically probable MSA-P is challenging in view of the lack of definite diagnostic tests to confirm the clinical suspicion of atypical parkinsonism. ${ }^{5}$ Neuropathological studies demonstrated that about $20 \%$ of patients clinically diagnosed as PD proved to have had another neurodegenerative disorder at postmortem examination and that signs and symptoms typical for the presence of PD (resting tremor, levodopa responsiveness) could also be found in necropsy verified MSA-P patients who were diagnosed in life as having $\mathrm{PD}^{6-8}$

In the past, functional brain imaging with positron emission tomography (PET) had been proved to be of some value for the differential diagnosis of parkinsonism. ${ }^{9-11}$ However, PET imaging was largely restricted to the measurement of striatal function. Because of the recent development of high resolution PET scanners and three dimensional magnetic resonance imaging (3D MRI) based methods of PET data analysis, extrastriatal cerebral regions can now also be investigated. In addition, 3D MRI volumetry has become available and has been recently applied in patients with PD, MSA-P, and progressive supranuclear palsy (PSP). ${ }^{12}$

The aim of this study was to evaluate whether the combined measurement of striatal and midbrain regions of interest as well as the use of two independent imaging modalities with multi-tracer PET and 3D MRI volumetry might further increase the diagnostic power in the distinction between PD and MSA-P. We hypothesised that extrastriatal histopathological changes in MSA-P might find correlates in these imaging modalities and focused on the midbrain as the extrastriatal region of choice, as a more extensive cell loss in the substantia nigra and locus coeruleus had been described in patients with MSA than in PD. ${ }^{13}{ }^{14}$

\section{METHODS}

\section{Study subjects}

Twenty four patients with PD diagnosed according to the UK brain bank criteria ${ }^{15}$ (18 men and 6 women, mean (SD) age 53.0 (11.0) years; mean (SD) Hoehn and Yahr ${ }^{16}$ (HY) score 1.9 (1.1); mean (SD) Unified Parkinson's Disease Rating Scale ${ }^{17}$

Abbreviations: $\mathrm{PET}$, positron emission tomography; MRI, magnetic resonance imaging; PD, Parkinson's disease; MSA; multiple system atrophy; FDG, [ $\left.{ }^{18} \mathrm{~F}\right]$ fluoro-deoxyglucose; RACLO, 11-C-Raclopride; VOI, volumes of interest; HY, Hoehn and Yahr score; UPDRS; Unified Parkinson's Disease Rating Scale; PSP progressive supranuclear palsy 
Table 1 Characteristics of the parkinsonian study subjects

\begin{tabular}{llll}
\hline & $\begin{array}{l}\text { Controls } \\
(\mathrm{n}=7)\end{array}$ & $\mathrm{PD}(\mathrm{n}=24)$ & MSA-P $(\mathrm{n}=9)$ \\
\hline Age & $53.4(11.9)$ & $53.0(11.0)$ & $56.1(5.4)$ \\
Disease duration & - & $5.2(4.7)$ & $4.9(2.7)$ \\
HY score & - & $1.9(1.1)$ & $3.6(0.5) \dagger$ \\
UPDRS total score & - & $35.3(27.2)$ & $66.4(15.4)^{*}$ \\
\hline
\end{tabular}

$P D$, idiopathic Parkinson's disease; MSA-P, striatonigral variant of multiple system atrophy. HY, Hoehn/Yahr Score; UPDRS, Unified Parkinson's Disease Rating Scale. ${ }^{*} p<0.005$ versus PD (ANOVA and post-hoc Scheffé test). $† p<0.0001$ versus PD (ANOVA and post-hoc Scheffé test). Data shown as mean (SD).

(UPDRS) 35.3 (27.2); mean (SD) disease duration 5.2 (4.7) years), nine patients with clinically probable MSA-P according to the criteria of Gilman et $a^{18}$ (two men and seven women, mean (SD) age 56.1 (5.4) years; mean (SD) HY score 3.6 (0.5); mean UPDRS 66.4 (15.4); mean (SD) disease duration 4.9 (2.7) years) as well as seven healthy controls (three men and four women, mean (SD) age 53.8 (10.0) years) were enrolled in the study. The MSA-P patients presented with progressive limb rigidity and bradykinaesia associated with early impairment of balance and gait. Dysarthria and urinary dysfunction was evident in six, postural hypotension in four, resting and action tremor, and ataxia and pyramidal signs in two further MSA-P subjects. Motor symptoms were universally refractory to levodopa and dopamine agonists. Family histories were negative for neurological and psychiatric disease in all patients, and there was no history of exposure to drugs or toxins, head injury, encephalitis, or metabolic disease. The healthy volunteers had no history of neurological diseases, nor any medication with central nervous system actions. The study was approved by the local ethics committee and informed consent was obtained from each individual. Demographic and clinical data of the study subgroups are presented in table 1. Clinical features of the MSA-P patients are summarised in table 2 .

\section{MRI imaging}

High resolution MRI was performed on a 1-Tesla Magnetom (Siemens AG, Erlangen, Germany) in a three dimensional fast low angle shot mode (echo time: $15 \mathrm{~ms}$; repetition time: $40 \mathrm{~ms}$; flip angle: 40 degrees), providing 64 contiguous slices with a pixel size of $0.98 \times 0.98 \mathrm{~mm}$ and a slice thickness of $2 \mathrm{~mm}$.

\section{PET imaging}

All subjects underwent multi-tracer positron emission tomography (PET) with 6-[ $\left.{ }^{18} \mathrm{~F}\right]$-fluoro-L-dopa (FDOPA), ${ }^{19}$ $\left[{ }^{18} \mathrm{~F}\right]$ fluoro-deoxyglucose (FDG), ${ }^{20}$ and 11-C-Raclopride $(\mathrm{RACLO})^{21}$ on consecutive days. The study subjects fasted overnight and had their antiparkinsonian medication withdrawn at least 16 hours before the PET scanning procedure.
Subjects laid comfortably in supine position in a room with dimmed lighting and low background noise. The PET scans were performed using the ECAT EXACT ${ }^{22}$ and ECAT EXACT HR positron scanner ${ }^{23}$ (Siemens CTI, Knoxville, TN, USA) yielding 47 contiguous transaxial image planes (slice thickness of $3.375 \mathrm{~mm}$ or $3.125 \mathrm{~mm}$, respectively, with a full width at half maximum axial resolution of 5.8 and $3.6 \mathrm{~mm}$ ). We used a 3D data acquisition mode to increase the sensitivity of the brains' signal to noise ratio, which obtains a higher spatial resolution and a reduction of the partial volume effect. Images were reconstructed on a $128 \times 128$ matrix with $2.17 \mathrm{~mm}$ pixel size.

\section{FDOPA}

A 100 mg carbidopa was administered orally one hour before PET scanning to inhibit the peripheral aromatic amino acid decarboxylase. A 10 minute transmission scan with a retractable ${ }^{68} \mathrm{Ga} /{ }^{68} \mathrm{Ge}$ ring source was performed to correct for tissue attenuation. All patients and controls received $370 \mathrm{Mbq}$ FDOPA intravenously. PET scanning began immediately after tracer injection following a protocol of nine time frames over 90 minutes.

\section{FDG}

Cerebral glucose metabolism was measured after injection of $370 \mathrm{MBq}$ of FDG. Regional cerebral metabolic rates of glucose (rCMRGlc) were calculated using the autoradiographic model with a fixed lumped constant of 0.42 and the rate constant $\mathrm{K}_{1}$ adjusted to measured tissue activity. ${ }^{20}$

\section{RACLO}

A bolus of $370 \mathrm{MBq}$ of ${ }^{11} \mathrm{C}$-raclopride (specific activity 30-70 $\mathrm{GBq} / \mu \mathrm{mol}$ ) was injected. Scanning began after a 30 second background frame recording a total of six serial time frames with 10 minute duration over a 60 minute study period.

\section{Data analysis}

Each MRI scan was reorientated three dimensionally to the anterior to posterior commissural (AC-PC) line, and exact 3D alignment of the PET scans with the MRI was obtained using a previously described coregistration procedure. ${ }^{24}$ The dataset of the reorientated MRI scan was subjected to a subtle analysis tool that permits the exact three dimensional definition of even small structures. ${ }^{25}$ The following volumes of interest (VOIs) were manually defined by outlining the appropriate anatomical structures in transverse slices of the individual $\mathrm{Tl}$ weighted MRI on both hemispheres: caudate nucleus, putamen (separated in two parts: anterior and posterior part), and midbrain. The upper border of the midbrain VOI was defined on the plane showing the mamillary bodies and the optic nerves, respectively, and the lower pontine border on the level of the middle cerebellar peduncle. After interactive VOI definition, their overall volumes were calculated by adding the volumes of all segmented voxels. Subsequently, corresponding volumes of both hemispheres were averaged. The advantage of

\begin{tabular}{|c|c|c|c|c|c|c|}
\hline Patient & Dysarthria & Tremor & Ataxia & $\begin{array}{l}\text { Pyramidal } \\
\text { signs }\end{array}$ & $\begin{array}{l}\text { Postural } \\
\text { hypotension }\end{array}$ & $\begin{array}{l}\text { Urinary } \\
\text { incontinence }\end{array}$ \\
\hline 1 & - & + & - & - & - & - \\
\hline 2 & + & - & - & - & + & - \\
\hline 3 & - & + & - & - & - & - \\
\hline 4 & - & - & - & - & + & + \\
\hline 5 & + & - & - & + & - & + \\
\hline 6 & + & - & - & - & + & + \\
\hline 7 & + & - & + & - & + & + \\
\hline 8 & + & - & - & - & - & + \\
\hline 9 & + & - & + & + & - & + \\
\hline
\end{tabular}




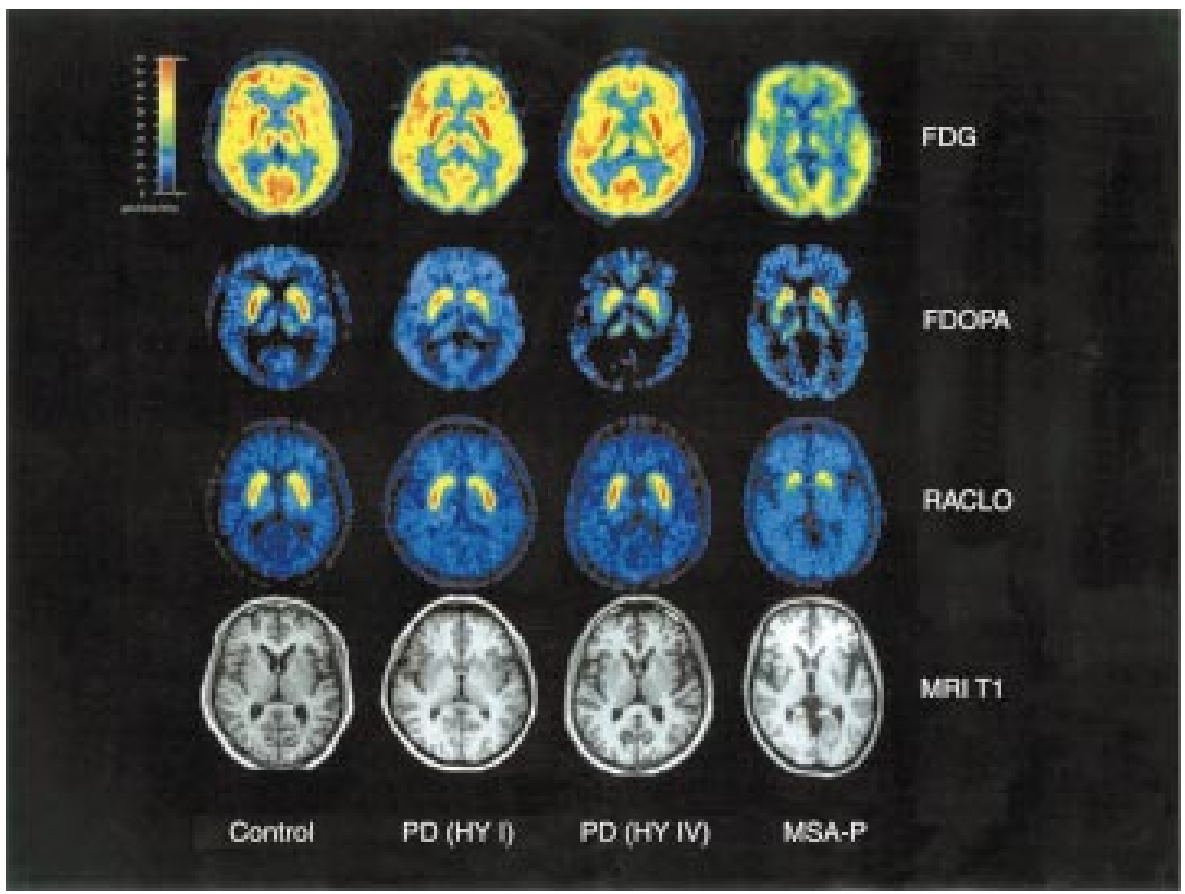

Figure 1 Transaxial slices of FDG-PET, FDOPA-PET, and RACLO-PET as well as T1 weighted MRI (from top to bottom) of a healthy control subject (control), two patients with idiopathic Parkinson's disease (early stage PD HY I and advanced stage PD HY IV), and a patient with striatonigral variant of multiple system atrophy (MSA-P) (from left to right). Note the marked decrease of putaminal volume (MRI), glucose consumption (FDG), and dopamine D2 receptor binding (RACLO) in MSA-P, which cannot be found in PD and controls. Reduction of putaminal FDOPA influx constants are similarly visible in PD and MSA-P with an intranuclear gradient of radiotracer binding towards lower acitivity within the posterior part of the putamen.

this type of data analysis is the exact definition of VOIs on the individual MRI scan for each patient as well as the formulation of MRI based volumetric data.

Using a fusion of individual PET images with the three dimensional VOI set, result values for the three different PET datasets were calculated for each VOI. FDOPA influx constants $\left(\mathrm{K}_{\mathrm{i}}\right)$, which represent the rate of striatal FDOPA uptake and storage, were calculated using a multiple time graphic analysis approach with an occipital instead of an arterial plasma function. ${ }^{26}$ Furthermore, we determined regional cerebral metabolic rates of glucose (rCMRGlc) for each VOI. ${ }^{20}$ To reduce intersubject variability, the rCMRGlc values were normalised by division with the global metabolic rate of glucose of the entire brain (gCMRGlc). ${ }^{27}$ Specific RACLO binding indices of the striatal VOIs were calculated with the cerebellum as dopamine-receptor free reference region using the following ratio index: (target VOI activity-cerebellum activity)/ cerebellum activity. ${ }^{2128}$ To capture local differences in putaminal function, we calculated a percentage binding gradient between the anterior and posterior part of the putamen for each PET tracer defined as the difference between both parts of the nucleus in relation to the activity of the entire putamen, which was set as $100 \%$. Negative values of the anterior-posterior gradient indicated more extensive decreases of the detected radioactivity within the posterior part of the putamen.

Statistical analyses were performed using SPSS for Windows 10.0 (SPSS UK Ltd, Surrey, England). Intergroup comparisons of metric data were performed with univariate analysis of variance (ANOVA) followed by Scheffé's test for correction of multiple comparisons. The level of statistical significance was set at $\mathrm{p}<0.05$.

\section{RESULTS}

\section{Clinical data}

The study subjects were well matched for age (table 1). Disease severity measured with the total UPDRS score was significantly higher in the MSA-P group (66.4 (15.4)) compared with PD subjects (35.3 (27.2)), whereas the disease duration was nearly identical in both patient groups (5.2 (4.7) years in PD versus 4.9 (2.7) years in MSA-P).

\section{Striatal imaging}

Compared with healthy controls, putaminal FDOPA $\mathrm{K}_{\mathrm{i}}$ values were significantly reduced in the MSA-P as well as in the PD group $(\mathrm{p}<0.0001)$ (table 3$)$. The decrease of FDOPA storage was mostly pronounced in the posterior putamen in both leading to a negative anterior-posterior gradient of radiotracer binding within this nucleus that was nearly identical in MSA-P (-46.8 (23.3)\%) and in PD subjects (-49.8 (22.4)\%). In both groups, the negative FDOPA gradient was significantly higher than in healthy controls $(-3.2(6.6) \%, \mathrm{p}<0.001)$ (fig l).

At the postsynaptic dopamine receptor level, MSA-P patients showed a significant reduction of all putaminal RACLO binding indices versus controls $(\mathrm{p}<0.05)$ and versus PD $(p<0.0001$; table 3$)$. The reduction of RACLO binding was most prominent in the posterior putamen, which resulted in a RACLO binding gradient of -19.8 (15.3)\% and mimicked the intranuclear anterior-posterior gradient of the FDOPA influx constants. In contrast, an increased RACLO uptake within the entire putamen was found in the PD cohort that reached the highest level in the posterior part of the nucleus (positive RACLO binding gradient of $+1.9(9.2) \%)$ and that was significantly higher compared with MSA-P subjects.

In MSA-P, the putaminal but not caudate rCMRGlc was significantly decreased compared with controls and PD patients (table 3). This finding was independent of the brains' global metabolic rate of glucose, as nearly identical statistically significant results were obtained after normalisation of the rCMRGlc values (table 3).

In MSA-P patients, the volumes of all putaminal VOIs were significantly reduced to approximately $50 \%$ of those of controls and PD patients (table 3). No significant striatal differences between PD patients and healthy controls were found in FDG-PET and MRI volumetry. 
Table 3 Results of striatal and midbrain PET imaging and 3D-MRI volumetry in seven healthy controls, 24 patients with $P D$, and nine subjects with MSA-P

\begin{tabular}{|c|c|c|c|}
\hline & Controls $(n=7)$ & $P D(n=24)$ & MSA-P $(n=9)$ \\
\hline \multicolumn{4}{|c|}{ FDOPA influx constants $\left(\mathrm{K}_{\mathrm{i}}\right)$} \\
\hline Caudate nucleus & $0.0105(0.0014)$ & $0.0085(0.0018)$ & $0.0081(0.0025)$ \\
\hline Putamen anterior & $0.0119(0.0012)$ & $0.0072(0.0023) \ddagger$ & $0.0058(0.0020) \ddagger$ \\
\hline Putamen posterior & $0.0115(0.0012)$ & $0.0044(0.0017) \ddagger$ & $0.0034(0.0011) \ddagger$ \\
\hline Putamen whole & $0.0116(0.0008)$ & $0.0061(0.0021) \ddagger$ & $0.0049(0.0016) \ddagger$ \\
\hline Midbrain & $0.0033(0.0006)$ & $0.0031(0.0008)$ & $0.0027(0.0013)^{\top}$ \\
\hline \multicolumn{4}{|l|}{ RACLO binding indices } \\
\hline Caudate nucleus & $1.54(0.22)$ & $1.76(0.38)$ & $1.31(0.49) \S$ \\
\hline Putamen anterior & $2.36(0.29)$ & $2.71(0.39)$ & $1.61(0.62)^{*} \|$ \\
\hline Putamen posterior & $2.03(0.21)$ & $2.79(0.54)^{*}$ & $1.20(0.87)^{*} \|$ \\
\hline Putamen whole & $2.25(0.21)$ & $2.76(0.42)^{*}$ & $1.45(0.68)^{*} \|$ \\
\hline \multicolumn{4}{|c|}{ FDG values (rCMRGlc) $(\mu \mathrm{mol} / 100 \mathrm{~g} / \mathrm{min})$} \\
\hline Caudate nucleus & $30.9(4.8)$ & $30.6(3.5)$ & $27.2(4.0)$ \\
\hline Putamen anterior & $39.6(4.0)$ & $38.2(5.5)$ & $29.9(4.3) *$ ขा \\
\hline Putamen posterior & $38.2(4.5)$ & $38.0(5.6)$ & $27.4(4.6) \dagger \|$ \\
\hline Putamen whole & $38.9(4.0)$ & $38.1(5.5)$ & $28.9(4.4)^{*} \|$ \\
\hline Midbrain & $23.4(2.2)$ & $23.1(2.4)$ & $22.6(2.2)$ \\
\hline \multicolumn{4}{|c|}{ Normalised FDG values (nrCMRGlc) } \\
\hline Caudate nucleus & $1.01(0.10)$ & $1.06(0.07)$ & $0.97(0.11)$ \\
\hline Putamen anterior & $1.30(0.06)$ & $1.32(0.10)$ & $1.07(0.16) \dagger \|$ \\
\hline Putamen posterior & $1.26(0.08)$ & $1.31(0.11)$ & $0.97(0.16) \ddagger \|$ \\
\hline Putamen whole & $1.28(0.06)$ & $1.31(0.10)$ & $1.03(0.16) \ddagger \|$ \\
\hline Midbrain & $0.77(0.09)$ & $0.80(0.07)$ & $0.80(0.05)$ \\
\hline \multicolumn{4}{|c|}{ 3D-MRI volumetry: regional volumes $\left(\mathrm{cm}^{3}\right)$} \\
\hline Caudate nucleus & $3.39(0.50)$ & $3.84(0.64)$ & $2.72(0.44) \|$ \\
\hline Putamen anterior & $2.58(0.44)$ & $2.73(0.40)$ & $1.41(0.43) \ddagger \|$ \\
\hline Putamen posterior & $1.65(0.23)$ & $1.70(0.23)$ & $0.78(0.26) \ddagger \|$ \\
\hline Putamen whole & $4.23(0.60)$ & $4.43(0.57)$ & $2.20(0.64) \ddagger \|$ \\
\hline Midbrain & $7.77(1.20)$ & $7.47(1.15)$ & $7.05(0.60)$ \\
\hline \multicolumn{4}{|c|}{ Putaminal anterior-posterior gradient } \\
\hline FDOPA & $-3.2(6.6)$ & $-49.8(22.4) \ddagger$ & $-46.8(23.2) \dagger$ \\
\hline RACLO & $-10.0(10.2)$ & $1.9(9.2)$ & $-19.8(15.3) \|$ \\
\hline FDG & $-3.8(7.5)$ & $-0.5(5.2)$ & $-9.0(5.6) \S$ \\
\hline
\end{tabular}

In figure 2, scatter plots of individual data are presented for the striatal PET and MR imaging modalities. In general, the caudate nucleus did not show a sufficient discrimination between the study subgroups in either modality. In FDOPAPET, we found a good discrimination between controls and parkinsonian subjects in the putamen but not between PD and MSA-P subjects (fig 2A). As the putaminal RACLO binding values were higher in $\mathrm{PD}$ than in controls, the dopamine receptor status differed adequately between the parkinsonian disorders but not between MSA-P and controls (fig 2B). Putaminal FDG-PET and MRI volumetry clearly separated MSA-P from PD and controls with the least overlap of individual data in MRI-volumetry and the posterior part of the nucleus (fig 2C and D).

\section{Midbrain imaging}

We found a small trend towards lower midbrain FDOPA $\mathrm{K}_{\mathrm{i}}$ values in both patient groups compared with healthy controls that amounted to $18 \%$ reduction of midbrain FDOPA uptake in MSA-P and to 6\% in PD subjects (table 3). However, these differences were not statistically significant. Neither absolute nor normalised midbrain rCMRGlc measured with FDG-PET differed significantly between the study subgroups. In MRI volumetry, we did not observe any significant intergroup differences either. However, a small 9\% reduction of the midbrain volume was found in the MSA-P group $(7.05(0.60) \mathrm{ccm})$ compared with controls $(7.77(1.20) \mathrm{ccm})$, which was not obvious in PD patients $(7.47$ (1.15) ccm, table 3$)$.

\section{DISCUSSION}

This study is the first to combine PET and 3D MRI based volumetric striatal and extrastriatal imaging in the same parkinsonian patients in order to differentiate between PD and MSA-P patients, which is often difficult on clinical findings alone. Our data suggest that putaminal RACLO-PET and FDG-PET as well as 3D MRI based putaminal volumetry are equivalent in their efficacy to distinguish PD from MSA-P patients, whereas functional and morphological imaging of the midbrain region does not provide a further gain in diagnostic accuracy.

The demonstration of putaminal signal alterations in MRI scans, such as putaminal atrophy, hyperintensive putaminal rim or hyperintensive putamen, might provide helpful information for the diagnosis of MSA, but is usually not sufficient because these changes are not specific to atypical parkinsonism. In a study published by Eidelberg and colleagues, these radiological signs were apparent only in four of eight MSA-P patients and not evident in a single patient who had MSA-P for less than five years. ${ }^{27}$ Schrag et al found routine MRI to fail in the differential diagnosis of PD and MSA-P in nearly $44 \%{ }^{30}$

In view of these shortcomings of standard MRI approaches, the volumetric assessment of striatal structures has been a subject of recent MRI based studies. Using the technique, Schulz and coworkers discriminated MSA-P from PD patients and normal controls, but not from MSA patients with predominant ataxia (MSA-C). ${ }^{12}$ We were able to clearly discriminate MSA-P patients both from PD and controls on the basis of striatal 3D MRI based volumetry, which proved a remarkebly reduced putaminal volume exclusively in MSA-P patients. The observed difference in MRI volumetry reached the same level of statistical significance as that found in FDGPET and RACLO-PET between PD and MSA-P subjects. Thus, putaminal abnormalities in MSA-P had been similarly detected with morphological and functional imaging modalities. 
A

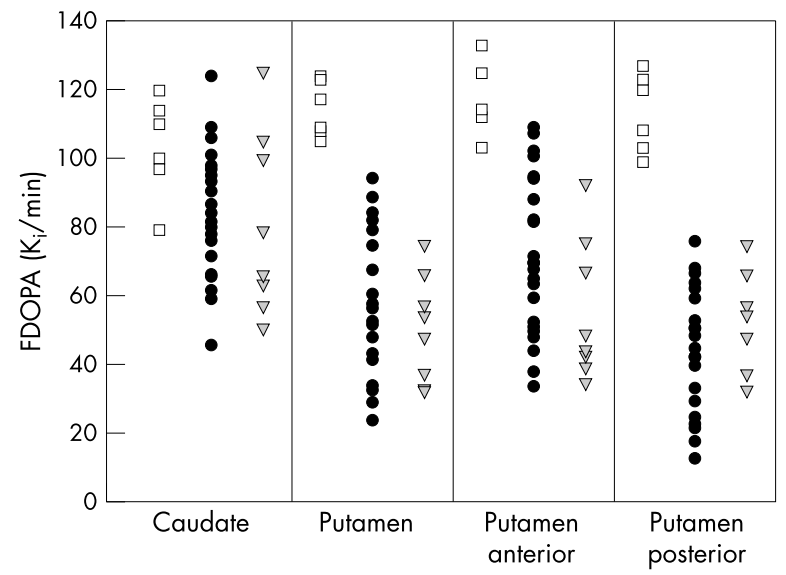

C

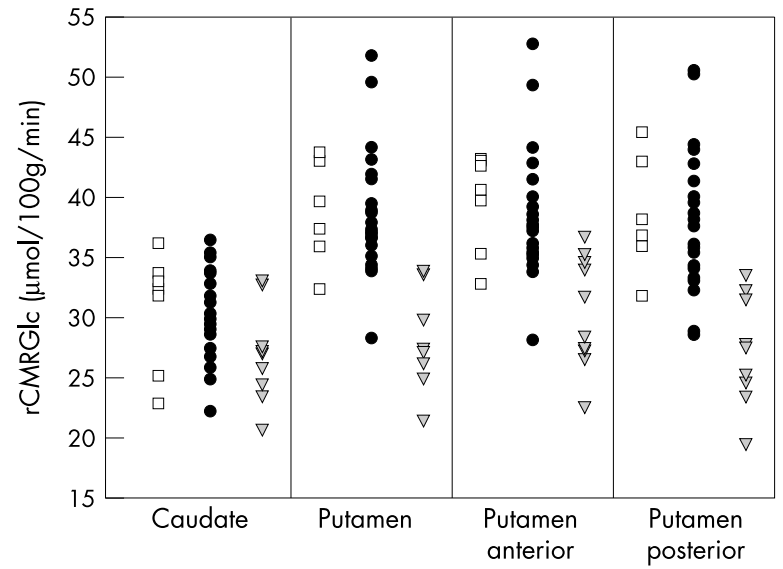

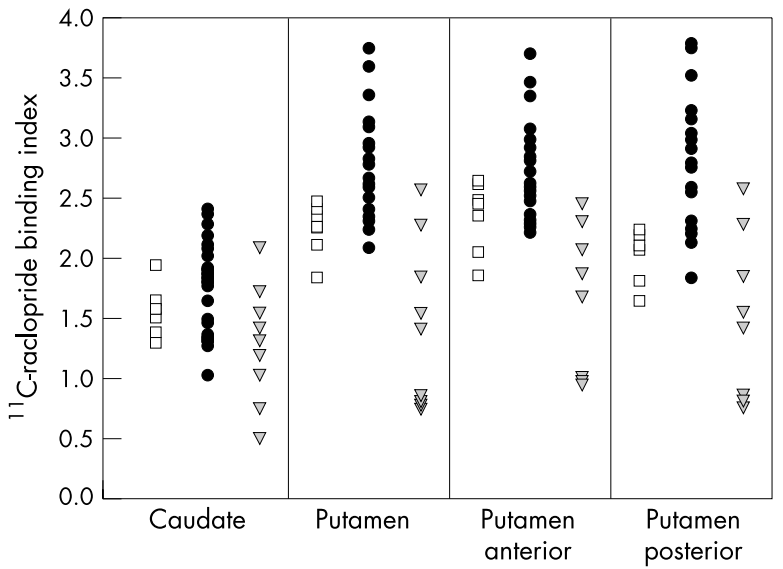

D

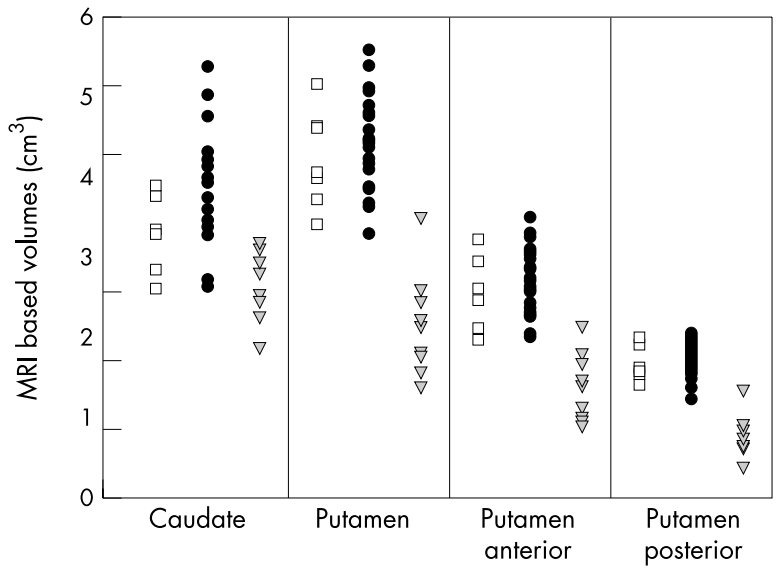

Figure 2 Scatter plots showing individual striatal PET (A; FDOPA, B: RACLO, C: FDG) and MRI based volumetry data (D) of controls (open squares), PD (black circles), and MSA-P study subjects (grey triangles). Within the putamen, a sufficient discrimination between controls and parkinsonian subjects as well as a substantial overlap between PD and MSA-P is obvious in FDOPA-PET. Putaminal RACLO binding is higher in PD than in controls and differentiated between the parkinsonian disorders, but not between MSA-P and controls. Putaminal FDG-PET and MRI volumetry clearly separated MSA-P from PD and controls with the least overlap of individual data in MRI volumetry and the posterior part of the nucleus.

Our PET data are in good agreement with previous findings in MSA and PD patients that showed decreases of the putaminal glucose metabolism and the postsynaptic dopamine receptor density exclusively in MSA, but not in levodopa responsive idiopathic PD. ${ }^{111-34}$ Histological and autoradiographic studies have shown that the levodopa resistance of motor symptoms as one key feature of MSA patients is associated with neuronal loss, gliosis, and loss of postsynaptic D2-dopamine receptors, preferentially in the posterior putamen. ${ }^{35}$ In a recent study, the application of diffusion weighted MRI imaging (DWI) in parkinsonian subjects discriminated PD from MSA-P patients by showing increased putaminal diffusion coefficients solely in MSA-P. ${ }^{36}$ This finding most probably reflects ongoing degeneration in MSA-P patients and corresponds to our putaminal FDG-PET and RACLO-PET findings. In contrast, we were able to demonstrate that the presynaptic dopaminergic deficit measured with FDOPA-PET does not differentiate between PD and MSA-P, as striatal FDOPA influx constants are reduced to a comparable degree and show an intranuclear gradient with lower values in the posterior part of the putamen in both disease entities. This finding is in line with histopathological data that found the most pronounced dopaminergic cell loss in the posterior part of the putamen in MSA patients. ${ }^{13}{ }^{14}$

The overlap of individual striatal imaging data in our study subjects suggests that MSA-P is most reliably separated from
PD by putaminal FDG-PET and MRI based volumetry data with the least overlap of values in the posterior part of the putamen. Therefore, the application of either imaging modality or their combination can be recommended for differential diagnostic purposes in case of atypical parkinsonism. While FDOPA-PET did not prove to be useful in the differentiation between MSA-P and PD, the putaminal RACLO binding also showed sufficient discrimination between slightly decreased tracer binding levels in MSA-P and remarkebly increased binding levels in PD. However, other groups also reported decreased striatal RACLO binding in advanced PD patients with a fluctuating response to levodopa medication. ${ }^{10}$ Therefore, the discrimination ability of RACLO-PET might be restricted, as it seems to strongly depend on clinical characteristics of PD patients.

With the use of PET, a reduction of striatal glucose consumption and of dopamine D2 receptor binding was also shown in patients with sporadic and familiar progressive supranuclear palsy (PSP), another important differential diagnosis of atypical parkinsonism. ${ }^{10}{ }^{37} 38$ In some patients, it is therefore difficult to decide whether the cause of atypical parkinsonism is MSA-P or PSP, even if striatal multi-tracer PET imaging is undertaken. To ensure reliability of our data, only patients with atypical parkinsonism fulfilling common diagnostic criteria of MSA-P $\mathrm{P}^{18}$ and without cardinal features of PSP, such as supranuclear vertical gaze palsy, predominant 
axial rigidity, and dementia, ${ }^{39}$ were included in the study. However, it has to be kept in mind that PET imaging or MRI volumetry is suitable to differentiate between PD and atypical parkinsonism, but has less diagnostic power to discriminate MSA-P from PSP, particularly in early disease stages in which a clinical differentiation is not yet obvious.

In the midbrain region, neither MRI volumetry nor PET imaging offered additional information for a better discrimination of PD and MSA-P. We did not expect this finding because, even though the distribution pattern of nigral degeneration may be similar in MSA-P and PD, neuronal loss has been shown to be more extensive in MSA than in PD in neuropathological studies. ${ }^{41}$ Further studies yielded evidence that the dopaminergic system is not the only one involved in MSA pathology. ${ }^{13}{ }^{14}$ For example, 5-hydroxytryptamine (5-HT) IB receptors were examined with the use of $[3 \mathrm{H}]$ sumatriptan as radioligand in posterior striatal and midbrain postmortem tissue sections of patients who died from various degenerative movement disorders. ${ }^{42}$ While no significant change in the density of $[3 \mathrm{H}]$ sumatriptan binding sites were found in the striatum and substantia nigra in PD patients, $[3 \mathrm{H}] \mathrm{su}-$ matriptan labelling was almost absent in MSA-P. Moreover, degeneration of the pontine noradrenergic locus coeruleus that may be involved in autonomic function and sleep-wakecycle regulation had been described in MSA and PD. ${ }^{13}{ }^{14}$

Despite the occurrence of a more extensive and multitransmitter affecting cell loss in MSA-P, we were not able to discriminate the parkinsonian study subgroups from each other and from healthy controls by means of midbrain PET imaging. The reasons for this finding remain speculative, as our study provides the first data on midbrain FDOPA activity in probable MSA-P patients and just a few studies are available on extrastriatal changes of FDOPA uptake in PD. ${ }^{43-45}$ In general, the cerebral FDOPA uptake mainly represents the intracellular enzymatic turnover of the radiotracer by the aromatic amino acid decarboxylase (AADC). ${ }^{46}$ As a common AADC enzyme is responsible for the decarboxylation of both levodopa in catecholaminergic and 5-hydroxytryptophan in serotonergic neurons, ${ }^{47}$ FDOPA-PET is not specific for dopaminergic neurons, but measures the activity of monoaminergic neurons in general. ${ }^{48}$ The midbrain as defined in our study included one dorsally located area comprising the serotonergic raphe nuclei and mesopontine segments with close topographical relations to catecholaminergic (locus coeruleus) as well as dopaminergic neurons (substantia nigra, retrorubral nucleus, ventral tegmental area). In previous studies performing voxel-by-voxel based analysis of FDOPA PET data with statistical parametric mapping, Ito et al found decreased $\mathrm{K}_{\mathrm{i}}$ values bilaterally in the substantia nigra of PD patients, ${ }^{49}$ whereas Rakshi and coworkers demonstrated significant increases of midbrain FDOPA activity in early, but significant reduction of midbrain FDOPA $\mathrm{K}_{\mathrm{i}}$ in advanced PD patients compared with controls. ${ }^{43}$ These apparently conflicting results indicate that the midbrain FDOPA PET signal presumably represents a complex summation of transmitter activity within monoaminergic neurons of the upper brainstem. It may be hypothesised that the PET signal reflects not only cell loss but also compensatory upregulation mechanisms in intact monaminergic neurons.

Similar to FDOPA, we failed to demonstrate significant differences in midbrain FDG-PET and in MRI volumetry in our study population. Presumably, MSA-P has its main pathological changes within the striatum and the substantia nigra, while infratentorial areas are more severely and earlier affected in MSA-C. De Volder et al studied seven patients with probable MSA-P and found a significantly decreased glucose utilisation in the putamen, caudate nucleus, and frontal cortex but not in the thalamus or cerebellum. ${ }^{34}$ Asato and coworkers showed that $76 \%$ of MSA-C, but only $44 \%$ of MSA-P patients had a pontine diameter smaller than the lower limit of the normal range. ${ }^{50}$ Therefore, imaging of the midbrain region might be of discriminant value in MSA-C, but shows no significant changes in MSA-P. However, another FDG-PET study showed similar levels of hypometabolism in the striatum as well as the cerebral cortex, thalamus, brainstem, and cerebellum in MSA and sporadic OPCA patients. ${ }^{33}$ Schulz and coworkers found a significant reduction of mean brainstem MRI volumes in patients with MSA-P and MSAC. ${ }^{12}$ However, with the exception of MSA-C, the individual brainstem volumes of MSA subjects showed an extensive overlap with the range of normal controls. Moreover, it has to be kept in mind that our data and the above cited MRI volumetric data are not directly comparable, as we focused our investigation on the rostral part of the brainstem, namely the midbrain region.

In conclusion, our data suggest that volumetric analysis of MRI data permits a clear distinction between PD and MSA-P and reports a considerable putaminal neuronal loss in MSA-P patients. The degeneration of striatal nuclei in MSA-P is also functionally reflected in a corresponding reduction of local glucose consumption estimated with FDG-PET and of postsynaptic dopamine-D2 receptor density measured with RACLOPET. However, striatal FDOPA influx constants as a measure of intact presynaptic dopaminergic nerve terminals, as well as MRI volumetry and PET imaging of the extrastriatal midbrain region, do not permit a distinction between the two disease entities. Our results indicate that both putaminal PET and MRI based volumetry may be considered equivalent in the differential diagnosis between PD and MSA-P. The alternative use of any diagnostic procedure can be recommended depending on its individual local availability.

\section{Authors' affiliations}

M Ghaemi, R Hilker, J Rudolf, J Sobesky, W-D Heiss, Neurology Department, University Hospital, Cologne, Germany W-D Heiss, Max-Planck-Institute for Neurological Research, Cologne, Germany

Competing interests: none declared.

\section{REFERENCES}

1 Jankovic J. Parkinsonism-plus syndromes. Mov Disord 1989;4 (suppl 1):95-119.

2 Wenning GK, Tison F, Ben-Shlomo Y, et al. Multiple system atrophy: a review of 203 pathologically proven cases. Mov Disord 1997; 12:133-47.

3 Adams RD, Van Bogaert L, Van der Eecken H. Striatonigral degeneration. J Neuropathol Exp Neurol 1964;23:584-608

4 Andrews JM, Terry RD, Spataro J. Striatonigral degeneration. Clinico-pathological correlations and response to stereotaxic surgery. Arch Neurol 1970;23:319-29.

5 Quinn N. Multiple system atrophy - the nature of the beast. J Neurol Neurosurg Psychiatry 1989;52 (suppl):78-89.

6 Hughes AJ, Colosimo C, Kleedorfer B, et al. The dopaminergic response in multiple system atrophy. J Neurol Neurosurg Psychiatry 1992;55:1009-13.

7 Colosimo C, Albanese A, Hughes AV, et al. Some specific clinical features differentiate multiple system atrophy (striatonigral variety) from Parkinson's disease. Arch Neurol 1995:52:294-8.

8 Ito $\mathbf{H}$, Kusaka H, Matsumoto S, et al. Striatal efferent involvement and its correlation to levodopa efficacy in patients with multiple system atrophy. Neurology 1996;47:1291-9.

9 Brooks DJ, Ibanez V, Sawle GV, et al. Differing patterns of striatal ${ }^{18} \mathrm{~F}$-dopa uptake in Parkinson's disease, multiple system atrophy, and progressive supranuclear palsy. Ann Neurol 1990;28:547-55.

10 Brooks DJ, Ibanez V, Sawle GV, et al. Striatal $D_{2}$ receptor status in patients with Parkinson's disease, striatonigral degeneration, and progressive supranuclear palsy, measured with 11 C-raclopride and positron emission tomography. Ann Neurol 1992;31:184-92.

11 Antonini A, Leenders KL, Vontobel P. Complementary PET studies of striatal neuronal function in the differential diagnosis between multiple system atrophy and Parkinson's disease. Brain 1997;120:2187-95.

12 Schulz JB, Skalei M, Wedekind D, et al. Magnetic resonance imaging-based volumetry differentiates idiopathic Parkinson's syndrome from multiple system atrophy and progressive supranuclear palsy. Ann Neurol 1999;45:65-74.

13 Oppenheimer D. Neuropathology of progressive autonomic failure. In: Bannister R, ed. Autonomic failure: a textbook of clinical disorders of the autonomic nervous system. Oxford: Oxford University Press, 1983:267-83. 
14 Gray F. The neuropathology of Parkinson syndrome. Rev Neurol 1988; 144:229-48.

15 Hughes AJ, Daniel SE, Kilford L, et al. Accuracy of clinical diagnosis of idiopathic Parkinson's diesease: a clinico-pathological study of 100 cases. J Neurol Neurosurg Psychiatry 1992;55:181-4.

16 Hoehn MM, Yahr MD. Parkinsonism: onset, progression and mortality. Neurology 1967; 17:427-42

17 Fahn S, Elton RL and the Members of the UPDRS Development Committee. Unified Parkinson's Disease Rating Scale. In: Fahn S, Marsden CD, Goldstein M, et al, eds. Recent developments in Parkinson's disease. Vol 2. New York, NY: MacMillan, 1987:153-63.

18 Gilman S, Low P, Quinn N, et al. Consensus statement on the diagnosis of multiple system atrophy. American Autonomic Society and American Academy of Neurology. Clin Auton Res 1998;8:359-62.

19 Garnett ES, Firnau G, Nahmias C. Dopamine visualized in the basal ganglia of living man. Nature 1983;305:137-8.

20 Wienhard K, Pawlik G, Herholz K, et al. Estimation of local cerebral glucose utilization by positron emission tomography of $\left[{ }^{18} \mathrm{~F}\right]$-2-fluoro-2-deoxy-D-glucose: a critical appraisal of optimization procedures. J Cereb Blood Flow Metab 1985;5:115-25.

21 Hume SP, Myers R, Bloomfield PM, et al. Quantification of 11 C-labeled raclopride in rat striatum using positron emission tomography. Synapse 1992; 12:47-54.

22 Wienhard K, Ericsson L, Grootoonk S, et al. Performance evaluation of the positron scanner ECAT EXACT. J Comput Assist Tomogr 1992;16:804-13.

23 Wienhard K, Dahlbom M, Eriksson L. The ECAT EXACT HR: performance of a new high resolution positron scanner. J Comput Assist Tomogr 1994;18:110-18.

24 Pietrzyk U, Herholz K, Fink GR, et al. An interactive technique for three-dimensional image registration: Validation for PET, SPECT, MRI and CT brain studies. J Nucl Med 1994;35:2011-18.

25 Stockhausen HM, Pietrzyk U, Herholz K, et al. A method for surface-based quantification of functional data from the human cortex. In: Carson E, Daube-Witherspoon ME, Herscovitch P, eds. Quantification functional brain imaging with positron emsission tomography. San Diego: Academic Press, 1998:139-42.

26 Patlak CS, Blasberg RG. Graphical evaluation of blood-to-brain transfer constants from multiple-time uptake data. Generalizations. J Cereb Blood Flow Metab 1985;5:584-90.

27 Eidelberg D, Moeller JR, Dhawan V, et al. The metabolic topography of Parkinsonism. J Cereb Blood Flow Metab 1994;14:783-801

28 Antonini A, Schwarz J, Oertel WH, et al. [ [ $\mathrm{C}$ C]raclopride and positron emission tomography in previously untreated patients with Parkinson's disease: influence of L-dopa and lisuride therapy on striatal dopamine $D_{2}$-receptors. Neurology 1994;44:1325-9.

29 Kaasinen V, Ruottinen HM, Nagren K, et al. Upregulation of putaminal dopamine $D_{2}$ receptors in early Parkinson's disease: A comparative PET study with [ $\left.{ }^{11} \mathrm{C}\right]$ Raclopride and [ $\left.{ }^{11} \mathrm{C}\right] \mathrm{N}-$ Methylspiperone. J Nucl Med 2000:41:65-70

30 Schrag A, Kingsley D, Phatouros C, et al. Clinical usefulness of magnetic resonance imaging in multiple system atrophy. J Neurol Neurosurg Psychiatry 1998;65:65-71.

31 Gilman S, Koeppe RA, Junk L, et al. Patterns of cerebral glucose metabolism detected with positron emission tomography differ in multiple system atrophy and olivopontocerebellar atrophy. Ann Neurol 1994;36: 166-75.
32 Kume A Takahashi A, Hashizume $Y$, et al. A histometrical and comparative study on Purkinje cell loss and olivary nucleus cell loss in multiple system atrophy. J Neurol Sci 1991;101:178-86.

33 Fulham MJ, Dubinsky RM, Polinsky RJ, et al. Computed tomography, magnetic resonance imaging and positron emission tomography with $\left[{ }^{18} \mathrm{~F}\right]$ fluorodeoxyglucose in multiple system atrophy and pure autonomic failure. Clin Auton Res 1991;1:27-36.

34 De Volder AG, Francart J, Laterrre C, et al. Decreased glucose utilization in the striatum and frontal lobe in probable striatonigral degeneration. Ann Neurol 1989;26:239-47.

35 Churchyard A, Donnan GA, Hughes A. Dopa resistance in multiple system atrophy: loss of postsynaptic D2 receptors. Ann Neurol 1993;34:219-26.

36 Schocke MFH, Seppi K, Esterhammer R, et al. Diffusion-weighted MRI differentiates the parkinson variant of multipe system atrophy from PD. Neurology 2002;58:575-80.

37 Piccini P, de Yebenz J, Lees AJ, et al. Detection of subclinical cases using $18 \mathrm{~F}-\mathrm{Dopa}$ and 18 Fluorodeoxyglucose positron emission tomography. Arch Neurol 2001;58:1846-51.

38 Karbe $\mathbf{H}$, Grond $M$, Huber $M$, et al. Subcortical damage and cortical dysfunction in progressive supranuclear palsy demonstrated by positron emission tomography. J Neurol 1992;239:98-102.

39 Steele JC, Richardson JC, Olszewski J. Progressive supranuclear palsy. Arch Neurol 1964;10:333-59.

40 Steele JC. Progressive supranuclear palsy. Brain 1972;95:693-704.

41 Fearnley JM, Lees AJ. Striatonigral degeneration: a clinicopathological study. Brain 1990;113:1823-42.

42 Castro ME, Pascual J, Romon T, et al. 5-HT1B receptor binding in degenerative movement disorders. Brain Res 1998;790:323-8.

43 Rakshi JS, Uema T, Ito K, et al. Frontal, midbrain and striatal dopaminergic function in early and advanced Parkinson's disease - A 3D $\left[{ }^{18} \mathrm{~F}\right]$ dopa-PET study. Brain 1999;122:1637-50.

44 Wuerker M, Ghaemi M, Baver A, et al. Changes of midbrain 18 dopa upake in different stages of Parkinson's disease. Mov Disord 1998;13 (suppl 2):42.

45 Ghaemi M, Rudolf J, Hilker R, et al. Increased pineal Fdopa uptake is related to severity of Parkinson's disease - A PET study. J Pineal Res 2001;30:213-19.

46 Firnau G, Sood S, Chirakal R, et al. Cerebral metabolism of 6- $\left[{ }^{18} \mathrm{~F}\right]$ fluoro-L-3,4-dihydroxy-phenylalanine in the primate. J Neurochem 1987:48:1077-82.

47 Tison F, Normand $E$, Jaber $M$, et al. Aromatic L-amino-acid-decarboxylase (DOPA decarboxylase) gene expression in dopaminergic and serotoninergic cells of the rat brainstem. Neurosci Lett 1991;127:203-6.

48 Brown WD, Taylor MD, Roberts AD, et al. FluoroDOPA-PET shows the non-dopaminergic as well as dopaminergic destinations of levodopa. Neurology 1999;53:1212-18.

49 Ito K, Morrish, PK, Rakshi JS, et al. Statistical parametric mapping with ${ }^{18} \mathrm{~F}$-dopa PET shows bilaterally reduced striatal and nigral dopaminergic function in early Parkinson's disease. J Neurol Neurosurg Psychiatry 1999;66:754-8.

50 Asato R, Akiguchi I, Masunaga S, et al. Magnetic resonance imaging distinguishes progressive supranuclear palsy from multiple system atrophy. J Neural Transm 2000;107: 1427-36. 